\title{
Understanding Phase Stability in Multilayers by Atom Probe Analysis
}

\author{
G.B. Thompson*, M.K. Miller*, R. Banerjee*, and H.L. Fraser* \\ *Department of Materials Science and Engineering, The Ohio State University, 2041 College Road, \\ Columbus, Ohio 43210 \\ ${ }^{* *}$ Oak Ridge National Laboratory, PO Box 2008, Oak Ridge, TN 37831-6136
}

Epitaxial monolithic thin films on substrates or individual layers in an A/B multilayered thin film stack can exhibit crystal structures that differ from the bulk equilibrium phase. The stabilization of these pseudomorphic phases is a result of the competition between the volumetric and interfacial free energies. Unlike a single film on a substrate, a multilayered thin film is unique in that each precedent layer can act as a template surface for the proceeding layer. Recently, a classical thermodynamic model has been properly extended to describe phase stability in an alternating A/B multilayer [1]. An outcome of this model has been a new type of phase diagram that depicts phase stability regions as a function of the inverse bilayer thickness, given as $\lambda^{-1}$, and the volume fraction, $f_{\mathrm{i}}$, of one of the constituent layers. The bilayer thickness, $\lambda$, is defined as the thickness of layer A plus layer $\mathrm{B}$. Thus the length scale, $\lambda$, and the volume fraction act as two independent degrees of freedom in stabilizing the pseudomoprhic phase. This is analogous to temperature and composition in traditional metallurgical phase diagrams. With the use of this model, a prediction of $h c p$ to $b c c$ phase stability for $\mathrm{Ti}$ in $\mathrm{Ti} / \mathrm{Nb}$ multilayers has been proposed.

The phase stability in Ti/Nb multilayers as a function $\lambda^{-1}$ and $f_{N b}$ is shown in Fig. 1 . The phases have been confirmed by x-ray and electron diffraction techniques. The experimentally determined phase stability boundary (solid line) in Fig. 1 is at a much lower layer thickness than the predicted boundary (dashed line). The slope of these boundaries can be derived from the classic thermodynamic model [1] to be equal to a ratio of the volumetric free energy difference, $\Delta G_{i}$ and the interfacial free energy reduction, $\Delta$, between the pseudomorphic and bulk states. Using a $\Delta G_{\mathrm{Ti}}$ from CALPHAD [2] and the measured slope of the boundary in Fig. 1, the experimentally determined $\Delta=-580 \mathrm{~mJ} / \mathrm{m}^{2}$. A van der Merwe interfacial energy model for a $b c c \mathrm{Ti} / b c c \mathrm{Nb}-$ $h c p \mathrm{Ti} / b c c \mathrm{Nb}$ interface predicted $\boldsymbol{\psi}=-280 \mathrm{~mJ} / \mathrm{m}^{2}[2]$.

Atom probe tomography is an ideal technique, due to its high spatial resolution, to understand the chemical profile across a nanostructured interface [5]. The associated thermodynamic free energies used to model the pseudomorphic phase stability are highly sensitive to the degree of intermixing at the interface and within each of the constituent layers. $\mathrm{Nb}$, being a $b c c$-stabilizer in Ti alloys, could have marked influence upon the phase stability of $b c c \mathrm{Ti}$ in the multilayer. A Ti/ $\mathrm{Nb}$ multilayer at the boundary $\left(\lambda=4.75 \mathrm{~nm}, f_{\mathrm{Nb}}=60 \%\right)$ has been prepared as an atom probe specimen in a similar manner to that by Martens et al. $[3,6]$. Three-dimensional atom probe maps and a compositional profile normal to the layers are shown in Figs. 2 and 3. It is evident from these figures that $\mathrm{Nb}$ has significantly interdiffused into the Ti layer to a concentration of $\approx 15 \mathrm{at} \%$. Refining the CALPHAD $\Delta G_{\mathrm{TiN} \text { ) }}$ with this $\mathrm{Nb}$ concentration coupled with the slope of Fig. 1, the experimental $\Delta y$ is approximately $-265 \mathrm{~mJ} / \mathrm{m}^{2}$; a value in closer agreement with the van der Merwe prediction. The interdiffusion of $\mathrm{Nb}$ into the Ti layers has significantly altered the free energies associated with stabilization of the pseudomorphic phase. One of the potential factors that could be contributing towards the enhanced interdiffusion of $\mathrm{Nb}$ into the $b c c$ Ti layers is the similar atomic radii of $\mathrm{Ti}(0.140 \mathrm{~nm})$ and $\mathrm{Nb}(0.145 \mathrm{~nm})$. Using a Matano construction, the interdiffusion coefficient across the Ti/Nb interface has been calculated from the compositional curves of Fig. 3 to be of the order $10^{-22} \mathrm{~cm}^{2} / \mathrm{s}$ [2]. Similar coefficient values have been reported in other thin film systems [4]. 


\section{References:}

[1] S.A. Dregia, R. Banerjee, and H.L. Fraser Scripta Mater. 29(2) (1998) 217.

[2] Gregory B. Thompson "Predicting Polymorphic Phase Stability in Multilayered Thin Films" Ph.D. Dissertation, Ohio State University (2003).

[3] R.L. Martens, D.J. Larson, T.F. Kelly, A.Cerezo, P.H. Clifton, and N. Tabat Microsc. Microanal. 6 (Supplement 2 Proceedings) (2000) 522.

[4] K.N. Tu Ann. Rev. Mater. Sci. 15 (1985) 147.

[5] Research at the Oak Ridge National Laboratory SHaRE Collaborative Research Center was sponsored by the Division of Materials Sciences and Engineering, U.S. Department of Energy, under contract DEAC05-00OR22725 with UT-Battelle, LLC.

[6] Cornell Nano-Scale Science \& Technology Facility (a member of the National Nanofabrication Users

Network) which is supported by the National Science Foundation under Grant ECS-9731293, its users, Cornell University and Industrial Affiliates.
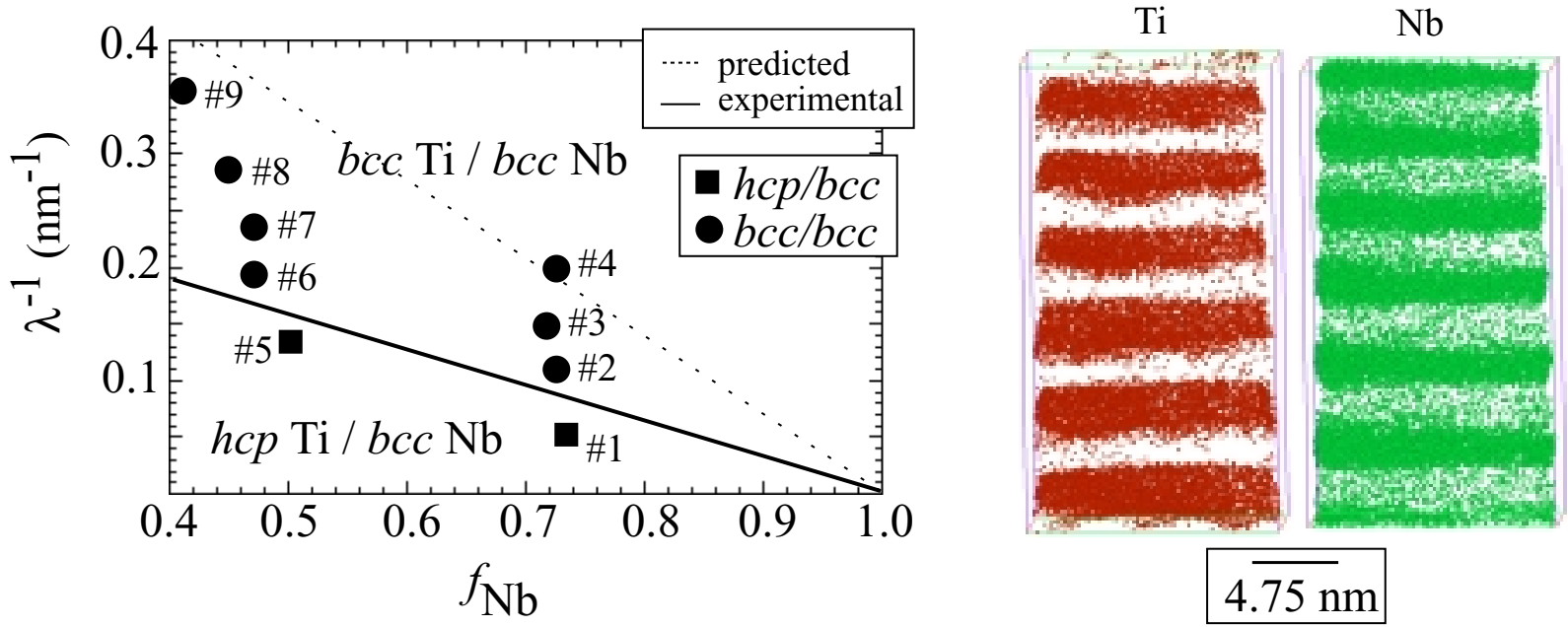

Fig. 1 Phase stability diagram for $\mathrm{Ti} / \mathrm{Nb}$ multilayers

Fig. 2 Reconstructed atom probe image of a Ti/Nb multilayer.

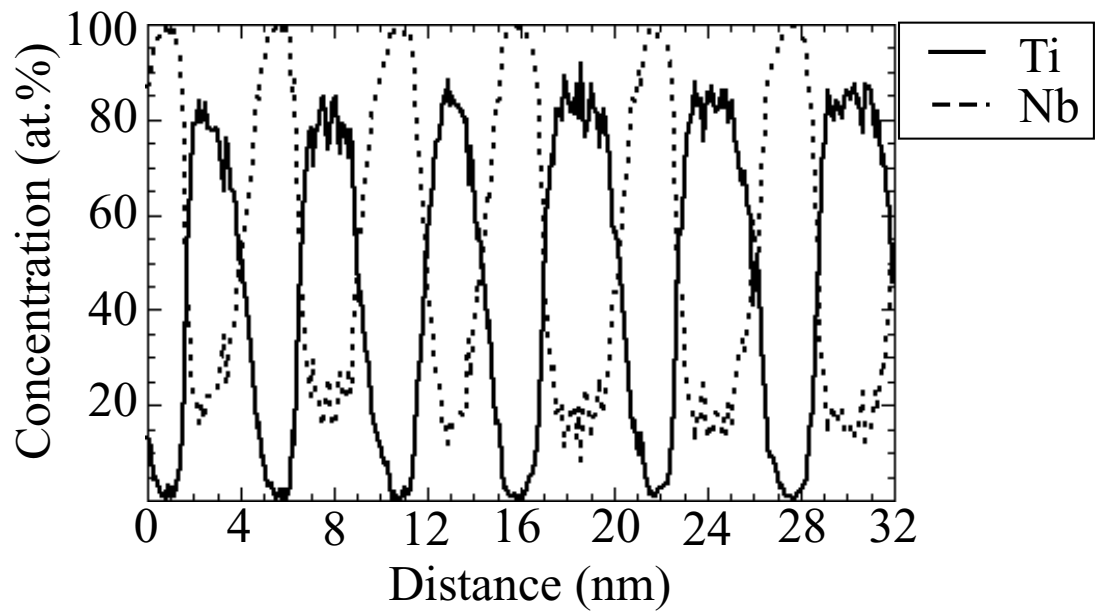

Fig. 3 Compositional profile normal to the interfaces of the $\mathrm{Ti} / \mathrm{Nb}$ multilayer of Fig. 2. 